\title{
Modelling the Effects of Antibiotics on Gut Flora Using a Nonlinear Compartment Model with Uncertain Parameters
}

\author{
Thulasi Jegatheesan and Hermann J. Eberl ${ }^{(凶)}$ \\ Department of Mathematics and Statistics, Biophysics Interdepartmental Program, \\ University of Guelph, Guelph, Canada \\ $\{$ tjegathe, heberl\}@uoguelph.ca
}

\begin{abstract}
We present a mathematical model of a microbial community in the human colon, including transport, exchange and metabolic processes. The colon is represented as a bioreactor with separate lumen and mucus microhabitats. The microbial community in the colon is grouped into four biomass functional groups based on their metabolic activity. While computational models present a challenge in the selection of model parameters, they also provide a more systematic approach to addressing uncertainties when compared to in vivo and in vitro experiments. We conduct an exploratory study on the uncertainty of our input parameters and a simulation study of the perturbation and recovery of gut microbiota from antibiotic exposure. We consider our parameters as random variables drawn from a uniform random distribution to reflect the diversity of gut microbial composition and variability between individuals.
\end{abstract}

Keywords: Mathematical model $\cdot$ Colon · Simulation experiments · Parameter uncertainty $\cdot$ ADM1

\section{Introduction}

The human intestine, or colon, is inhabited by a diverse and dynamic microbial community that harbours over 1000 types of microbes [8]. This microbial community plays an important role in human health and has a mutualistic relationship with the host [16]. The intestinal microbiota are responsible for many functions, such as maintaining the gastrointestinal epithelial barrier, preventing pathogens from adhering to intestinal surfaces, and the development of the immune system [17]. A core function of the gut microbiota is carbon fermentation and the breakdown of complex carbohydrates into short chain fatty acids (SCFAs) and gas. The conversion of these carbohydrates into SCFAs contributes to human energy requirements, regulation of intestinal physiology, and immune function [9].

Supported by Natural Sciences and Engineering Research Council of Canada (NSERC): RGPIN-2019-05003, RTI-2016-00080. 
The composition of gut microbial communities varies between individuals and may be altered by many factors such as diet, genetics, antibiotic treatment, and environmental factors $[3,6,8]$. Many in vivo studies have shown that an imbalance in gut microbiota composition through loss of microbial and metabolic diversity can result in the development of disease [15]. Due to host-specific gut community composition, as well as confounding environmental factors and lack of easy access to the gut, it is difficult to draw conclusions about underlying function and behaviour of the gut microbiota [19]. In vitro experiments aim to provide insight by experimentally simulating the gut using a bioreactor. These experiments are more economical and allow for greater experimental control when compared to human studies, however repeated experiments can be expensive and it is difficult to exactly replicate the physiology of the colon, particularly the mucus layer. Additionally, microbial community composition can vary between bioreactor models due to the source of the inocula [20], making it difficult to draw population-level conclusions. In silico, or computational models can be used as a predictive and explanatory tool for biological processes and phenomena. They can allow for the mechanistic investigation of the gut microbiota while overcoming several limitations of in vivo and in vitro studies. They eliminate the ethical considerations of in vivo studies, can incorporate interactions and processes that are not possible in vitro, and repeated in silico simulations are more economical than in vivo trials and in vitro experiments.

Presented in this paper is a mechanistic model of the microbiota in the human colon that considers the lumen and mucus environments of the colon, microbial interactions, and transport and exchange processes within the colon. Despite the large inter-individual variation between gut microbial communities, core metabolic pathways are conserved across individuals due to functional redundancy in microbial groups [8]. Rather than representing individual species of microbes, gut microbiota can be organized based on their metabolic activity into biomass functional groups (BFGs). Each BFG is responsible for a particular metabolic pathway based on substrate preference and fermentation products. This is modelled using mass balance equations for biomass growth, substrate consumption and product formation. There are a number of studies that model the gut microbiota using mass balance equations with varying degrees of complexity. The review by Williams et al. [19] includes models that range from a single-species in a simple chemostat reactor to multi-species models with complex physical representations of the colon. Several models are based on the Anaerobic Digestion Model No. 1 (ADM1) [1], which is a generalised model of anaerobic digestion, typically applied to wastewater. The ADM1 was modified by [14] to consider only the degradation of carbohydrates to glucose, lactate, hydrogen, carbon dioxide, water, methane and SCFAs (acetate, propionate, butyrate). Since the majority of substrates available for fermentation in the human colon consist of carbohydrates, [14] excludes the digestion of proteins and lipids, acid-base reactions in ADM1. Many current mechanistic models of anaerobic digestion in the gut are adaptions and extensions of the carbohydrate model in [14], modified to include more realistic physical representations of the colon $[7,11]$, digestion 
of proteins and lipids $[4,5]$ and further subdivision of BFGs $[4,11]$. Our model uses the metabolic processes in [11] with a simplified physical representation of the colon. A bacteriostatic antibiotic treatment which inhibits the growth of the microbiota is included. The subsequent recovery of microbiota after perturbation is investigated with probiotics and prebiotics.

Complex models lead to more realistic representations of biological systems but increased complexity often limits possible theoretical analyses. Complex mathematical models typically require computational simulations with large parameter spaces. A limitation of mechanism-based and predictive modeling often stems from the difficulties in model parameter estimation, since experimental data is needed to validate and calibrate model behaviour. These models require clear estimates of stoichiometric and kinetic properties of model processes in order to make meaningful predictions. However, bioreactor operating conditions such as temperature, $\mathrm{pH}$ and source of inocula determine the composition of bioreactor microbial communities and can result in variability in the measured parameters. Due to the diversity of gut microbial composition, there is an inherent variability of parameters between individuals. A specific set of parameters could be taken to represent the microbial composition and physiology of a particular individual. As such, the uncertainty of the parameter space must be considered when drawing conclusions from simulation results of computational models of the gut, to account for both variability in measured experimental parameters and inter-individual variability in gut microbiota composition. In [4], each BFG includes multiple strains with kinetic parameters drawn randomly from a uniform distribution. We consider all model parameters, including kinetic and exchange parameters, as a uniform random variables to account for the variability in gut microbiota diversity and physiological variability between individuals.

\section{Model Formulation}

\subsection{Reactor Representation}

The human colon can be described as an anaerobic bioreactor. Previous work has described the human colon as a single-stage reactor $[4,10,13]$, a three-stage reactor $[10,14]$ and a plug-flow reactor $[7,11,12]$. In this study, to capture the lumen and mucus microhabitats, the colon is modelled as a continuous stirredtank reactor (CSTR) with a lateral diffusion compartment (see Fig.1). The main (lumen) compartment, has inflow from the upper gastrointestinal tract (GIT) and outflow out of the colon as well as removal of water and metabolites from the lumen into the host. There is no inflow and outflow from the lateral diffusion (mucus) compartment but there is exchange between the lumen and mucus compartments. In a typical system, fiber is the only inflow into the lumen compartment and mucins are endogenously produced in the mucus compartment. The flow rate through the lumen compartment is assumed to be continuous and is chosen such that washout of biomass does not occur. 


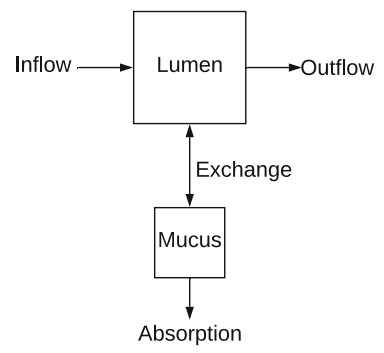

Fig. 1. Reactor representation of the gut with inflow from the upper GIT, outflow out of the colon and exchange between the mucus and lumen compartments

\section{$2.2 \quad$ Reaction Kinetics}

In both the lumen and mucus compartment, anaerobic digestion of carbohydrates is carried out by intestinal microbiota. Rather than characterising individual species, microbiota are categorised into four biomass functional groups (BFGs) based on their metabolic activity: sugar degrading biomass (SD), lactate degrading biomass (LD), acetogenic biomass (HDA), and methanogenic biomass (HDM). Due to the redundancy in metabolic pathways in the colon, five main reaction pathways are considered (Fig. 2), each facilitated by a biomass functional group (BFG). Fiber and mucin are assumed to be the only fermentable carbohydrates available to the microbiota. Fiber enters the lumen compartment at a constant rate from the upper GIT and mucin is produced endogenously by the host. The SD are the only BFG that are able to degrade the fiber and mucin into consumable monomer sugars. The hydrolysis of fiber and mucin into monomer sugars by $\mathrm{SD}$ is based on Contois kinetics $\left(\phi_{1}(\boldsymbol{c})\right.$ in Table 1$)$. Monod kinetics is applied to the fermentation of sugar by SD, lactate by LD, acetogenesis by HDA and methanogenesis by HDM $\left(\phi_{2}(\boldsymbol{c})-\phi_{5}(\boldsymbol{c})\right.$ in Table 1$)$.

The entirety of the reaction processes are shown in Table 1 . The rows correspond to the processes and the columns correspond to the components. The components consist of the biomass and metabolites and are associated with the lumen of mucus microhabitat that they occupy. The reaction rates are given by $\phi_{j}(\boldsymbol{c})$ and include growth kinetics and decay of biomass. Endogenous mucin production is modelled as in [11] and is a source of fiber in the mucus compartment.

\subsection{Exchange}

The exchange of materials between the lumen and mucus compartment can be categorised into passive and active exchange. Sugar is the only component to passively diffuse between compartments. In general, all other components are exchanged through attachment (lumen to mucus), detachment (mucus to lumen), and absorption (mucus to host). 


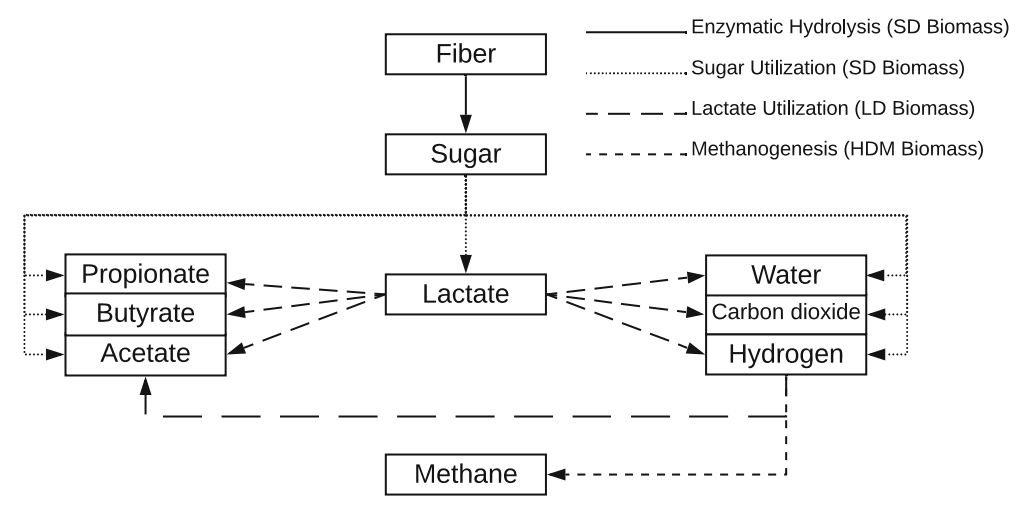

Fig. 2. Metabolic reaction pathways showing the degradation of fiber into short chain fatty acids and gas by the four biomass functional groups

\subsection{Complete Model}

The matrix representation of the complete model including transport, exchange and reaction kinetics is given by:

$$
\frac{d}{d t}\left(\begin{array}{c}
C_{L} \\
C_{M}
\end{array}\right)=\left(\begin{array}{cc}
S^{T} & 0 \\
0 & S^{T}
\end{array}\right)\left(\begin{array}{l}
\phi\left(C_{L}\right) \\
\phi\left(C_{M}\right)
\end{array}\right)-\left(\begin{array}{cc}
-D_{L L} & D_{L M} \\
D_{M L} & -D_{M M}
\end{array}\right)\left(\begin{array}{l}
C_{L} \\
C_{M}
\end{array}\right)+\left(\begin{array}{c}
u_{L} \\
u_{M}
\end{array}\right)
$$

The variables $C_{L}$ and $C_{M}$ are each vectors of length 14 and contain the concentrations of biomass, substrates and products in the lumen and mucus, respectively. $S^{T}$ is the transpose of the stoichiometric matrix in Table 1 and is of size $14 \times 9 . \phi\left(C_{L}\right)$ and $\phi\left(C_{M}\right)$ are the reaction rates given in Table 1 and are each

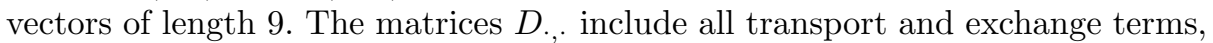
are non-negative and diagonal and are of dimension $14 \times 14$. The vectors $u_{L}$ and $u_{M}$ are each of length 14 and contain the inflow of substrate, biomass of products into the system. In a typical simulation, the only external input into the system is a fiber inflow to the lumen compartment. Since fiber is the only input into the system, $u_{L}$ contains only one non-zero entry. $u_{M}$ is a vector of zeros as there is no external input into the mucus compartment.

\subsection{Antibiotics Mechanism of Action}

A bacteriostatic antibiotic is considered in the simulation studies. The antibiotic mechanism is designed to elicit a nonlethal response from the microbiota by preventing the proliferation of bacteria. Antibiotic action is modelled as a growth inhibition term such that biomass growth is inhibited as antibiotic concentration increases. The growth inhibition term is written as $\frac{K_{A}}{K_{A}+A}$, where $A$ is the concentration of antibiotic. In the antibiotic simulation experiments, the input concentration of the antibiotic is constant and held at $A=1$. As a result, the parameter $K_{A}$ term solely controls the strength of the antibiotic. Only single continuous doses of finite duration of antibiotics are considered in the following simulation study. 
Table 1. Stoichiometric matrix, $S$, of reaction processes. This matrix $S$ and the reaction rates $\phi$ correspond to Eq. (1) in Sect. 2.4

\begin{tabular}{l}
\multicolumn{2}{l}{ For soluble components } \\
\hline
\end{tabular}

\section{Results of Simulation Experiments}

\subsection{Exploration of Parameter Space}

The presented model involves a large multi-dimensional parameter space. In order to increase our confidence in the qualitative predictions of the model, we used a brute force approach to investigating the sensitivity of potentially influential parameter sets. Considering the parameters as random variables, simulations were conducted in replicate, drawing parameters from a uniform random distribution prior to the start of the simulation. The parameters were held constant over the course of a simulation. Default parameter values from the literature are taken as mean values. This approach is used to ensure that the observed qualitative behaviour is typical for a reasonable parameter range. If any parameter sets significantly impact the longterm behaviour of the system, they may require extra consideration in simulation studies. Simulations were performed in replicates of 100 with a parameter upper and lower bound of $\pm 10 \%$ the mean 
value unless otherwise specified. With limited information about the distributions of model parameters, a uniform random distribution was chosen to reflect species heterogeneity with BFGs, host heterogeneity in species composition and to account for variability in bioreactor setups. The uniform distribution allows for parameters to be selected in only a biologically relevant (nonzero) range. The equilibrium concentration of the biomass species, particularly the sugar degrading biomass, are compared to the default equilibrium values to determine the sensitivity of the system to a parameter set.

The default parameters used for simulations were obtained from [11], which were in turn adapted from $[1,14]$. In general, the parameters that most influence the behaviour of the system can be classified as reaction parameters, which control substrate consumption and biomass growth, and exchange parameters, which control the exchange of materials between the lumen and mucus compartments. Both sets can be determined empirically, with the variability of reaction parameters being more well-studied experimentally. The yield coefficients $(Y$ in Table 1) are derived from the balanced chemical equations describing the conversion of reactants to products and are not varied in this study.

Four preliminary studies were conducted to investigate the sensitivity of the system to the reaction and exchange parameters. The parameter sets are as follows: (1) $10 \%$ uncertainty on all reaction parameters performed in 200 replicates, (2) $50 \%$ uncertainty on all reaction parameters performed in 200 replicates, (3) $10 \%$ uncertainty on exchange parameters performed in 100 replicates, and (4) $10 \%$ uncertainty on exchange parameters responsible for the movement of mucins and sugar between compartments in 100 replicates. The sensitivity of the output was determined using the variance of the steady state values of the SD since they are at the top of the metabolic hierarchy and highly influence the behaviour of the other three BFGs. For the reaction parameters, the distribution of steady state values of the lumen SD biomass had a standard deviation of 0.219 and 4.965 for an uncertainty of $10 \%$ and $50 \%$ respectively. The distribution of steady state values of the mucus SD biomass had a standard deviation of 1.264 and 27.899. Figures 3 and 4 shows the distribution of the steady state values for the sugar degrading biomass with an uncertainty of $10 \%$ and $50 \%$. The sensitivity of the output to exchange parameters similarly shows a small variance in SD steady state values for an input uncertainty of $10 \%$ on the exchange parameter set. The distributions of steady state values for the full set of exchange parameters and the subset of top level exchange parameters are shown in Figs. 5 and 6.

\subsection{Calibrating Antibiotic Strength}

Model output was found to be relatively stable in response to uncertainty in reaction and exchange parameters. Since these parameters did not have a significant effect on model output, they were held at their default values for the following simulation studies. As a result, simulating the effects of antibiotics on the gut microbiota only requires the consideration of the parameter $K_{A}$ and the duration of antibiotic administration. Simulations were conducted by selecting a $K_{A}$ value within a biologically relevant range. Small values of $K_{A}$ result in 

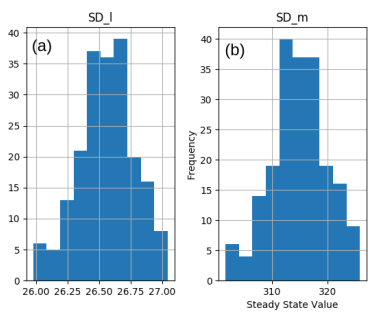

Fig. 3. Distribution of sugar degrading biomass steady state values in lumen (a) and mucus (b) with uncertainty of $10 \%$ for reaction and exchange parameters
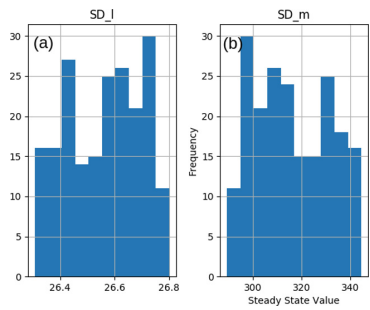

Fig. 5. Distribution of sugar degrading biomass steady state values in lumen (a) and mucus (b) with uncertainty of $10 \%$ for top level exchange parameters

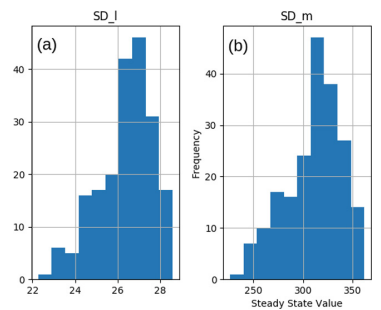

Fig. 4. Distribution of sugar degrading biomass steady state values in lumen (a) and mucus (b) with uncertainty of $50 \%$ for reaction and exchange parameters
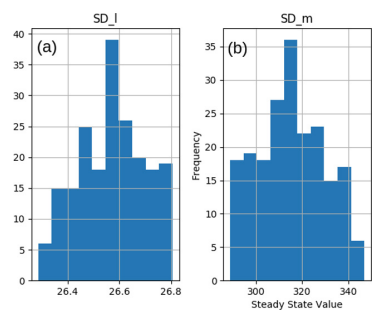

Fig. 6. Distribution of sugar degrading biomass steady state values in lumen (a) and mucus (b) with uncertainty of $10 \%$ for all exchange parameters

eradication of the population (Figs. 7, 8, 9 and 10) or too drastic of an effect (Figs. 11, 12, 13 and 14) with an antibiotic administered for 14 days.

A $K_{A}$ value of 60 with a duration of 14 days results in a moderate perturbation of the biomass with a recovery to equilibrium values (Figs. 15, 16, 17 and 18). This value of $K_{A}$ with a dose period of 14 days is used in the following simulation study as the baseline antibiotic strength. At this strength, the antibiotic effect in the lumen and mucus are similar with the SD biomass being affected the most. This is followed by the LD biomass, with little effect on the methanogens and acetogens. The SCFAs and substrates are not heavily impacted.

\subsection{Mitigation Strategies and Recovery Time}

Antibiotic use can shift gut microbiota composition from a healthy to an unhealthy equilibrium that can persist for years [8]. Prebiotics and probiotics are often used to alleviate the side effects of antibiotics that result from the loss of gut microbiota. A simulation study was carried out to investigate the effect of different antibiotic mitigation strategies in the form of prebiotics and probiotics. Prebiotics, which are a 'dietary component that fosters the growth of beneficial bacteria' [18] were considered in the form of a single dose of fiber 


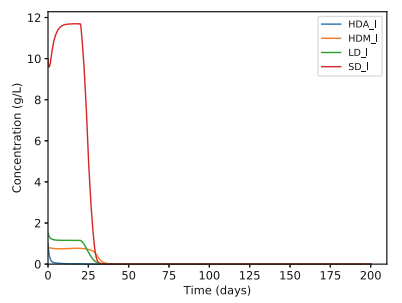

Fig. 7. Lumen concentrations of biomass with 14 day dose of lethal antibiotic, $K_{A}$ $=1.35$

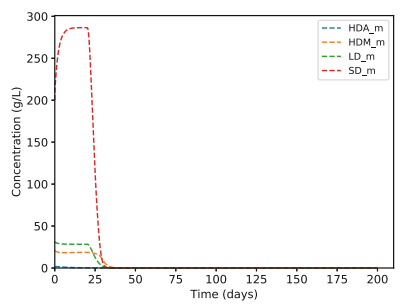

Fig. 9. Mucus concentrations of biomass with 14 day dose of lethal antibiotic, $K_{A}$ $=1.35$

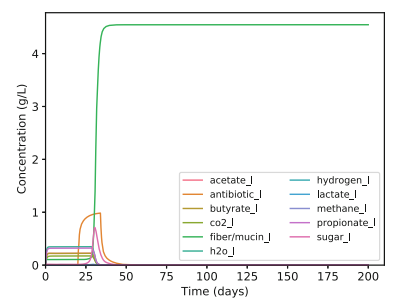

Fig. 8. Lumen concentrations of metabolites with 14 day dose of lethal antibiotic, $K_{A}=1.35$

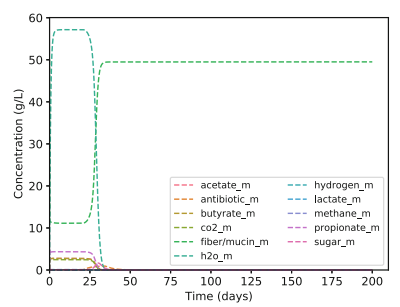

Fig. 10. Mucus concentrations of metabolites with 14 day dose of lethal antibiotic, $K_{A}=1.35$

or sugar before or after antibiotic treatment. Probiotics, which are live microorganisms that provide a health benefit to the host $[2,18]$, were in the form of a discrete input of either all BFGs or only sugar degrading biomass before or after antibiotic treatment. A 14 day dose with a weak antibiotic is investigated, as well as shorter doses of stronger antibiotics. The antibiotic strength $K_{A}$ and the antibiotic dose duration are adjusted such that the total amount of antibiotic administered remains the same for all dosing regimes. The prebiotics and probiotics are administered at a constant input into the system for 7 days. Recovery time of each simulation is measured as the time from the end of the antibiotic dose to the time when the biomass has reached $99 \%$ of the equilibrium values prior to antibiotic perturbation. A treatment is classified as effective if the percent difference in recovery time decreases for at least one biomass species when compared to the recovery time with no treatment. Tables 2 and 3 summarize the efficacy of the different treatments and dosing regimes. For both prebiotic and probiotic treatments, administration during the antibiotic dosing reduced recovery time most consistently. Sugar was a more effective prebiotic treatment when compared to fiber, and a probiotic with only sugar degrading biomass was more effective than a complete probiotic. It was also consistently easier to mitigate the effects of an antibiotic administered at moderate strength for a moderate amount of time when compared to a long course weak antibiotic. It was however not possible to decrease recovery time for strong antibiotics that were 


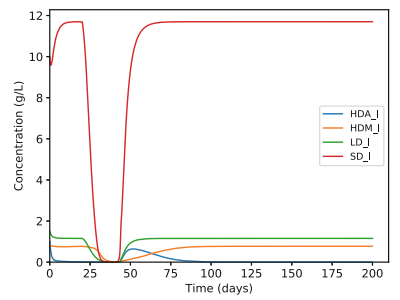

Fig. 11. Lumen concentrations of biomass with 14 day dose of strong antibiotic, $K_{A}=1.45$

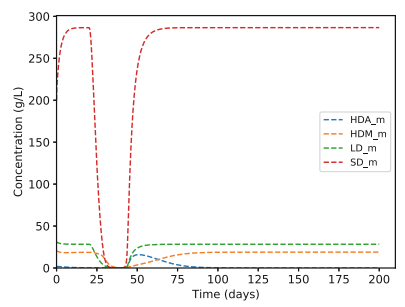

Fig. 13. Mucus concentrations of biomass with 14 day dose of strong antibiotic, $K_{A}=1.45$

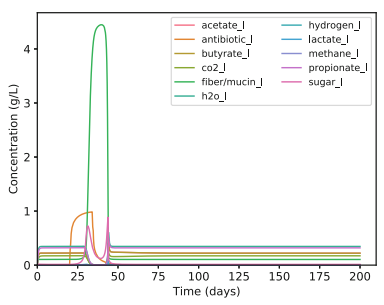

Fig. 12. Lumen concentrations of metabolites with 14 day dose of strong antibiotic, $K_{A}=1.45$

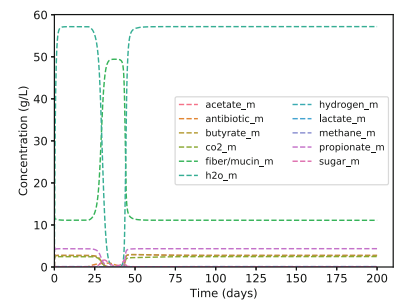

Fig. 14. Mucus concentrations of metabolites with 14 day dose of strong antibiotic, $K_{A}=1.45$

administered over less than a day. In all cases, prebiotic and probiotic treatment had little effect on substrate and product concentrations. Figures 19 and 20 show the percent difference in recovery time for the most effective treatment.

Table 2. Summary of effectiveness of prebiotics for recovery of the communities in lumen and mucus compartments for different regimes. Each row represents one simulation, with the X's indicating the treatment regime

\begin{tabular}{l|l|l|l|l|l|l|l}
\hline $\begin{array}{l}\text { Antibiotic } \\
\text { short dose }\end{array}$ & $\begin{array}{l}\text { Antibiotic } \\
\text { moderate dose }\end{array}$ & $\begin{array}{l}\text { Antibiotic } \\
\text { long dose }\end{array}$ & $\begin{array}{l}\text { Before } \\
\text { antibiotic }\end{array}$ & $\begin{array}{l}\text { During } \\
\text { antibiotic }\end{array}$ & $\begin{array}{l}\text { Sugar } \\
\text { prebiotic }\end{array}$ & $\begin{array}{l}\text { Fiber } \\
\text { prebiotic }\end{array}$ & Efficacy \\
\hline $\mathrm{X}$ & & & $\mathrm{X}$ & & & $\mathrm{X}$ & None \\
\hline & $\mathrm{X}$ & $\mathrm{X}$ & $\mathrm{X}$ & & $\mathrm{X}$ & $\mathrm{X}$ & None \\
\hline $\mathrm{X}$ & & & $\mathrm{X}$ & & $\mathrm{X}$ & None \\
\hline & & $\mathrm{X}$ & $\mathrm{X}$ & $\mathrm{X}$ & & $\mathrm{X}$ & Both \\
\hline $\mathrm{X}$ & & & $\mathrm{X}$ & $\mathrm{X}$ & & None \\
\hline & & $\mathrm{X}$ & $\mathrm{X}$ & & $\mathrm{X}$ & & Bone \\
\hline $\mathrm{X}$ & & $\mathrm{X}$ & $\mathrm{X}$ & & $\mathrm{X}$ & & Mucus \\
\hline & & $\mathrm{X}$ & & $\mathrm{X}$ & $\mathrm{X}$ & & None \\
\hline & & $\mathrm{X}$ & & $\mathrm{X}$ & $\mathrm{X}$ & & Both \\
\hline
\end{tabular}




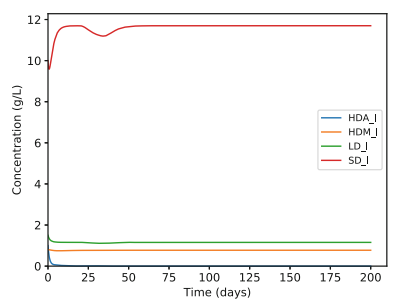

Fig. 15. Lumen concentrations of biomass with 14 day dose of moderate strength antibiotic, $K_{A}=60$

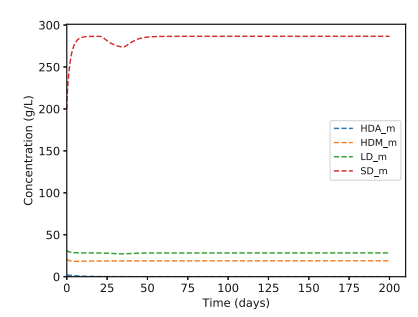

Fig. 17. Mucus concentrations of biomass with 14 day dose of moderate strength antibiotic, $K_{A}=60$

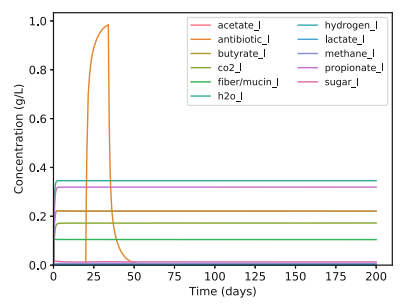

Fig. 16. Lumen concentrations of metabolites with 14 day dose of moderate strength antibiotic, $K_{A}=60$

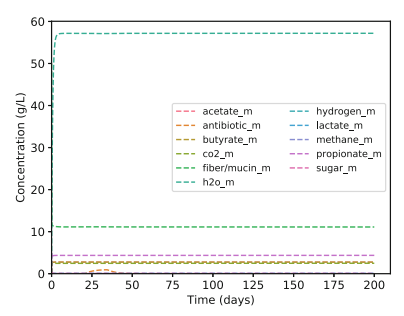

Fig. 18. Mucus concentrations of metabolites with 14 day dose of moderate strength antibiotic, $K_{A}=60$

Table 3. Summary of effectiveness of probiotics for recovery of the communities in lumen and mucus compartments for different regimes. Each row represents one simulation, with the X's indicating the treatment regime

\begin{tabular}{l|l|l|l|l|l|l|l}
\hline $\begin{array}{l}\text { Antibiotic } \\
\text { short dose }\end{array}$ & $\begin{array}{l}\text { Antibiotic } \\
\text { moderate dose }\end{array}$ & $\begin{array}{l}\text { Antibiotic } \\
\text { long dose }\end{array}$ & $\begin{array}{l}\text { Before } \\
\text { antibiotic }\end{array}$ & $\begin{array}{l}\text { During } \\
\text { antibiotic }\end{array}$ & $\begin{array}{l}\text { SD } \\
\text { probiotic }\end{array}$ & $\begin{array}{l}\text { Complete } \\
\text { probiotic }\end{array}$ & Efficacy \\
\hline $\mathrm{X}$ & $\mathrm{X}$ & $\mathrm{X}$ & $\mathrm{X}$ & & $\mathrm{X}$ & $\mathrm{X}$ & None \\
\hline & & $\mathrm{X}$ & $\mathrm{X}$ & & $\mathrm{X}$ & Lumen \\
\hline $\mathrm{X}$ & & & $\mathrm{X}$ & & $\mathrm{X}$ & None \\
\hline & $\mathrm{X}$ & & $\mathrm{X}$ & $\mathrm{X}$ & & $\mathrm{X}$ & Both \\
\hline $\mathrm{X}$ & & & $\mathrm{X}$ & $\mathrm{X}$ & & $\mathrm{X}$ & Lumen \\
\hline & $\mathrm{X}$ & & $\mathrm{X}$ & & $\mathrm{X}$ & & None \\
\hline $\mathrm{X}$ & & $\mathrm{X}$ & $\mathrm{X}$ & & $\mathrm{X}$ & & Both \\
\hline & $\mathrm{X}$ & & & $\mathrm{X}$ & $\mathrm{X}$ & & None \\
\hline & & $\mathrm{X}$ & & $\mathrm{X}$ & $\mathrm{X}$ & & Bone \\
\hline
\end{tabular}




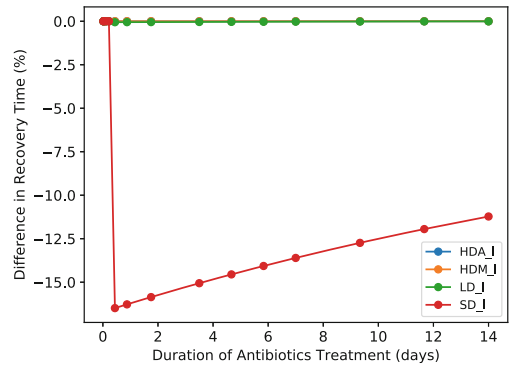

Fig. 19. Lumen concentrations of biomass after treatment with sugar degrading probiotic during antibiotic dosing

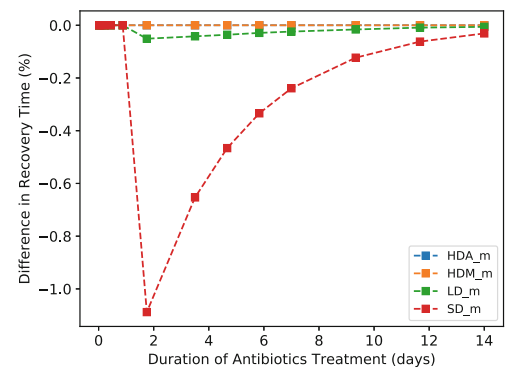

Fig. 20. Mucus concentrations of biomass after treatment with sugar degrading probiotic during antibiotic dosing

\subsection{Uncertainty in Antibiotic Parameter $K_{A}$}

To confirm whether the difference in recovery time between prebiotic/probiotic treatment and untreated antibiotic exposure is statistically significant, we conducted a simulation study to compare two regimes of antibiotics, with $K_{A}=60$ and $K_{A}=30$ as default values and an antibiotic course of 14 days. A subset of prebiotic and probiotic treatments are repeated with randomly generated $K_{A}$ and growth parameters using the method described in Sect.3.1. See Table 4 for a summary of the results. The recovery time with no prebiotic or probiotic treatment is taken as the base value for comparisons. A t-test is used to determine whether the recovery time with prebiotic/probiotic treatment with uncertainty on $K_{A}$ and growth parameters is statistically different when compared to the recovery time with no treatment. Treatment recovery times with p-values less than 0.05 are considered significantly different from recovery times with no treatment. Treatment regimes where there is no improvement in recovery time using default parameter values also show no improvement in recovery time in the sample set.

Table 4. Summary of Effectiveness of Probiotics for Different Regimes

\begin{tabular}{l|l|l|l|l|l|l}
\hline Simulation & $K_{A}$ & Base & Population mean & Sample mean & Std. dev & p-value \\
\hline Pre. before & 60 & 11.23 & 5.409 & 5.465 & 0.1815 & 0.00263 \\
Pre. before & 30 & 14.58 & 8.677 & 8.754 & 0.2697 & 0.00523 \\
Pre. during & 60 & 11.22 & 2.075 & 2.088 & 0.1056 & 0.228 \\
Pre. during & 30 & 14.53 & 4.820 & 4.842 & 0.09125 & 0.0229 \\
\hline Pro. before & 60 & 11.23 & 0.0 & 0.0 & 0.0 & N/A \\
Pro. before & 30 & 14.58 & 0.0 & 0.0 & 0.0 & N/A \\
Pro. during & 60 & 11.23 & 0.0 & 0.0 & 0.0 & N/A \\
Pro. during & 30 & 14.53 & 4.753 & 4.748 & 0.09334 & 0.573 \\
\hline
\end{tabular}




\section{Conclusions}

Uncertainty of parameters is an inherent part of computational models, particularly in physiological models with heterogeneity across individuals. Including parameter uncertainty as an intrinsic part of computational experimental design allows for model outcomes to be used in a predictive or explanatory capacity on a population level. For many computational models there is an underlying idea that there is a true set of parameter values. However, for many biological models, parameter values may exist within a range. For the model presented in this study, treating the parameters as random variables helps to address the diversity of microbial gut composition and variability within and between individuals. In this preliminary computational study, we find that variability in parameters do not have a significant impact on long-term model outcomes. A more thorough analysis of the parameter distribution and uncertainty of the input parameters is necessary to draw further conclusions.

Theoretical analyses of mathematical models can provide insight into system behaviour. It is often difficult or not possible to carry out this type of analysis for large and complex models. Due to the hierarchical structure of the metabolic processes in our system, the long-term survival of the gut microbiota are dependent on the survival of the sugar degrading biomass group, which is responsible for the degradation of carbohydrates into usable monomer sugars. Antibiotic mitigation strategies were found to be most effective if they targeted this group by supplementing their population with a probiotic or their diet with a prebiotic. Other applications of this model to human health and disease, such as the role of SCFAs may require the consideration of the full system. However, in the context of recovery after antibiotic treatment, the top level of our system can be studied independently. A reduced system would consist of the equations for fiber, mucin and SD biomass, corresponding to rows 1, 2 and 6 in Table 1 . This reduced system allows for theoretical analysis and results in a reduced parameter space. Studying this system could aid in the design of computational studies of the full system, particularly in the scope of microbial recovery after antibiotic perturbation.

\section{References}

1. Batstone, D.J., et al.: The IWA anaerobic digestion model no 1 (ADM1). Water Sci. Technol. 45(10), 65-73 (2002)

2. Hickson, M.: Probiotics in the prevention of antibiotic-associated diarrhoea and Clostridium difficile infection. Ther. Adv. Gastroenterol. 4(3), 185-197 (2011)

3. Huttenhower, C., et al.: Structure, function and diversity of the healthy human microbiome. Nature 486(7402), 207 (2012)

4. Kettle, H., Petra, L., Holtrop, G., Duncan, S.H., Flint, H.J.: Modelling the emergent dynamics and major metabolites of the human colonic microbiota. Environ. Microbiol. 17(5), 1615-1630 (2015)

5. Kettle, H., Holtrop, G., Louis, P., Flint, H.J.: microPop: modelling microbial populations and communities in R. Methods Ecol. Evol. 9(2), 399-409 (2018) 
6. Kolde, R., et al.: Host genetic variation and its microbiome interactions within the Human Microbiome Project. Genome Med. 10(1), 6 (2018)

7. Labarthe, S., Polizzi, B., Phan, T., Goudon, T., Ribot, M., Laroche, B.: A mathematical model to investigate the key drivers of the biogeography of the colon microbiota. J. Theor. Biol. 462, 552-581 (2019)

8. Lozupone, C., Stomabaugh, J., Gordon, J., Jansson, J., Knight, R.: Diversity, stability and resilience of the human gut microbiota. Nature 489(7415), 220-230 (2012)

9. Marchesi, J.R., et al.: The gut microbiota and host health: a new clinical frontier. Gut 65(2), 330-339 (2016)

10. Moorthy, A.S., Eberl, H.J.: Assessing the influence of reactor system design criteria on the performance of model colon fermentation units. J. Biosci. Bioeng. 117(4), 478-484 (2014)

11. Moorthy, A.S., Brooks, S.P.J., Kalmokoff, M., Eberl, H.J.: A spatially continuous model of carbohydrate digestion and transport processes in the colon. PLoS One 10(12), 1-17 (2015)

12. Moorthy, A.S., Eberl, H.J.: compuGUT: an in silico platform for simulating intestinal fermentation. SoftwareX 6, 237-242 (2017)

13. Motelica-Wagenaar, A.M., Nauta, A., van den Heuvel, E.G., Kleerebezem, R.: Flux analysis of the human proximal colon using anaerobic digestion model 1. Anaerobe 28, 137-148 (2014)

14. Muñoz-Tamayo, R., Laroche, B., Walter, E., Doré, J., Leclerc, M.: Mathematical modelling of carbohydrate degradation by human colon microbiota. J. Theor. Biol. 266(1), 189-201 (2010)

15. Reid, G., Younes, J.A., Van Der Mei, H.C., Gloor, G.B., Knight, R., Busscher, H.J.: Microbiota restoration: natural and supplemented recovery of human microbial communities. Nat. Rev. Microbiol. 9(1), 27-38 (2011)

16. Rowland, I., et al.: Gut microbiota functions: metabolism of nutrients and other food components. Eur. J. Nutr. 57(1), 1-24 (2017). https://doi.org/10.1007/ s00394-017-1445-8

17. Sánchez, B., Delgado, S., Blanco-Míguez, A., Lourenço, A., Gueimonde, M., Margolles, A.: Probiotics, gut microbiota, and their influence on host health and disease. Molec. Nutr. Food Res. 61(1), 1-15 (2017)

18. Sartor, B.R.: Therapeutic manipulation of the enteric microflora in inflammatory bowel diseases: antibiotics, probiotics, and prebiotics. Gastroenterology 126(6), 1620-1633 (2004)

19. Williams, C.F., Walton, G.E., Jiang, L., Plummer, S., Garaiova, I., Gibson, G.R.: Comparative analysis of intestinal tract models. Ann. Rev. Food Sci. Technol. 6, 329-350 (2015)

20. Yen, S., et al.: Metabolomic analysis of human fecal microbiota: a comparison of feces-derived communities and defined mixed communities. J. Proteome Res. 14(3), 1472-1482 (2015) 